\title{
Superior Dislocation of the Patella: Case Report and Literature Review
}

\author{
Paul E. Caldwell*, Samuel Carter and Sara E. Pearson \\ Orthopedic Research of Virginia (SC, PEC and SEP) and Tuckahoe Orthopedic Associates, Ltd., (PEC), USA
}

Submission: December 20, 2016; Published: January 09, 2017

*Corresponding author: Paul E. Caldwell III MD, 1501 Maple Avenue, Suite 200, Richmond, VA 23226, Ph: (804) 527-5960;

Fax: (804) 527-5961; Email: pcaldwell50@hotmail.com

\section{Abstract}

A 46-year-old female presented to the emergency department with a rare superior dislocation of the patella. Magnetic resonance imaging revealed inferior osteophytes on the patella engaging osteophytes on the superior portion of the trochlear groove resulting in a locked superior patellar dislocation. A closed reduction was performed, resulting in immediate pain relief and nearly full active range of motion.

Keywords: Superior patellar dislocation; Closed reduction; Non operative treatment

\section{Case Report}

A 46-year-old female presented to the emergency department (ED) with complaints of significant anterior knee discomfort, swelling and inability to ambulate or actively flex her knee. She reported falling at home and striking her right knee directly on a piano stool. She denied any history of injury to the right knee and reported only occasional mild anterior knee pain with kneeling or squatting prior to this accident. Initial examination in the ED showed a prominent and superiorly displaced patella. There was a visible and palpable dimple inferior to the patella that appeared to be a defect in the patellar tendon. There was a small effusion, and she was unable to flex her knee or perform a straight leg raise. Any attempt at quadriceps activation caused significant pain.

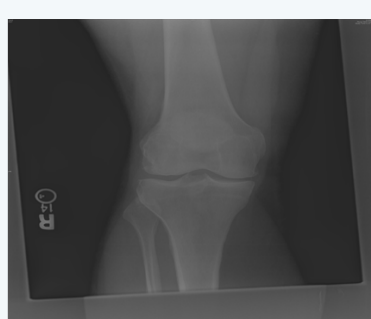

$1 \mathrm{~A}$

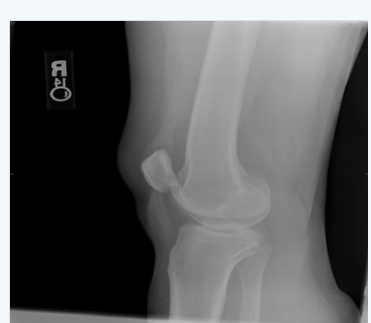

$1 B$
Figure 1A: Anteroposterior (AP).

Figure 1B: lateral $X$-rays taken in the emergency department (ED) demonstrating superior displacement of the patella without fracture and an unusual anterior tilt.
X-rays (Figure 1) taken in the ED demonstrated superior displacement of the patella without fracture and an unusual anterior tilt of the patella. The initial diagnosis in the ED was a patellar tendon rupture, and a magnetic resonance image (MRI) of the right knee was performed after orthopedic consultation. The MRI (Figure 2) confirmed that the patellar tendon and the remainder of the extensor mechanism were intact. It also demonstrated that inferior osteophytes on the patella were engaged onto osteophytes on the superior portion of the trochlear groove resulting in a locked superior patellar dislocation.

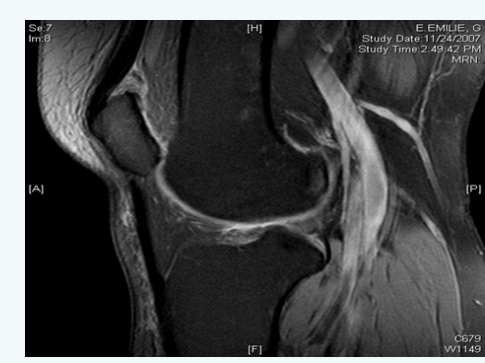

Figure 2: Magnetic resonance image (MRI) confirming that the patellar tendon and the remainder of the extensor mechanism were intact. Inferior osteophytes on the patella were engaged onto osteophytes on the superior portion of the trochlear groove, resulting in a locked superior patellar dislocation.

The patient was given intravenous sedation in the ED and a closed reduction was performed by applying gentle superiorly 


\section{Orthopedics and Rheumatology Open Access Journal}

directed pressure on the patella, with subsequent unlocking of the osteophytes. The patient had immediate relief of her discomfort and was able to perform a straight leg raise and had nearly full active flexion after the reduction. She was placed in a knee immobilizer for comfort and was discharged home. She was examined in the clinic after one week and demonstrated painless full active range of motion and has had no further squeal from this episode at 18-month follow-up.

\section{Discussion}

Superior dislocation of the patella is a rare event. There have been over 20 reports of superior patellar dislocation in the literature, the first of which was reported by Watson-Jones [1] in 1956. Nearly all of these cases occurred in middle-aged or elderly patients with degenerative changes in the patellofemoral joint and involved interlocking osteophytes. There was one report of superior dislocation in a 34-year-old rugby player with no evidence of patellofemoral arthrosis [2]. The mechanism of injury is consistently either a posterior- or upwardly-directed force on the inferior pole of the patella, as was the case in our patient when she struck her patella on a piano stool, or active quadriceps contraction with hyperextension of the knee.

Superior dislocation of the patella can clinically mimic a patellar tendon rupture and thus can initially present some difficulty in diagnosis and management in the emergency room setting. In both situations the patient cannot perform a straight leg raise. With dislocation, however, the patellar tendon is intact and under tension so the patient has difficulty actively or passively flexing the knee as well. The superior pole of the patella will always be tilted anteriorly with a superior dislocation, due to the fixed position of the inferior pole and the tension in the tendon. An MRI is often helpful to evaluate the integrity of the patellar tendon and distinguish between these two diagnoses [3].

Treatment usually consists of closed reduction with adjuncts ranging from simple analgesia to general anesthesia. Yip et al. [4] proposed immobilization at $30^{\circ}$ in a flexed knee brace immediately after reduction to prevent a recurrence as the patient is recovering from anesthesia. There is controversy regarding the necessity of arthroscopic osteoplasty to remove the offending osteophytes. Since our patient along with the majority of patients reviewed in the literature recovered uneventfully without repeated locking episodes, we recommend conservative management for this uncommon occurrence. Arthroscopy is generally reserved for a patient with recurrent dislocations or mechanical symptoms or those patients that can voluntarily dislocate [5].

\section{Acknowledgement}

The following entities have provided grant funding to Orthopaedic Research of Virginia for Fellowship support: Arthrex, Bon Secours, DJO, DePuy-Mitek, and Smith \& Nephew.

\section{Conflict of Interest}

None of the authors nor members of their families have received financial remuneration related to the subject of the article.

\section{References}

1. Watson Jones R (1974) Fractures and joint injuries. ( $5^{\text {th }}$ edn). Baltimore: Williams \& Wilkins. Clin Orthop Relat Res 105: 4-10.

2. Saleemi AJ, Hussain A, Iqbal MJ, Thuse MG, George AA (2007) Superior dislocation of patella in a rugby player: An update on a extremely rare condition and review of the literature. Knee Surg Sports Traumatol Arthros 15: 1112-1113.

3. Iorwerth A, Thomas R, Shewring DJ (2001) Confirmation of an intact patellar tendon in superior dislocation of the patella using magnetic resonance imaging. Injury 32(2): 167-169.

4. Yip DK, Wong JW, Sun LK, Wong NM, Chan CW, et al. (2004) The management of superior dislocation of the patella with interlocking osteophytes: An update on a rare problem. J Orthop Surg 12(2): 253257.

5. Takai S, Yoshino N, Hirasawa Y (1998) Arthroscopic treatment of voluntary superior dislocation of the patella. Arthroscopy 14(7): 753756.

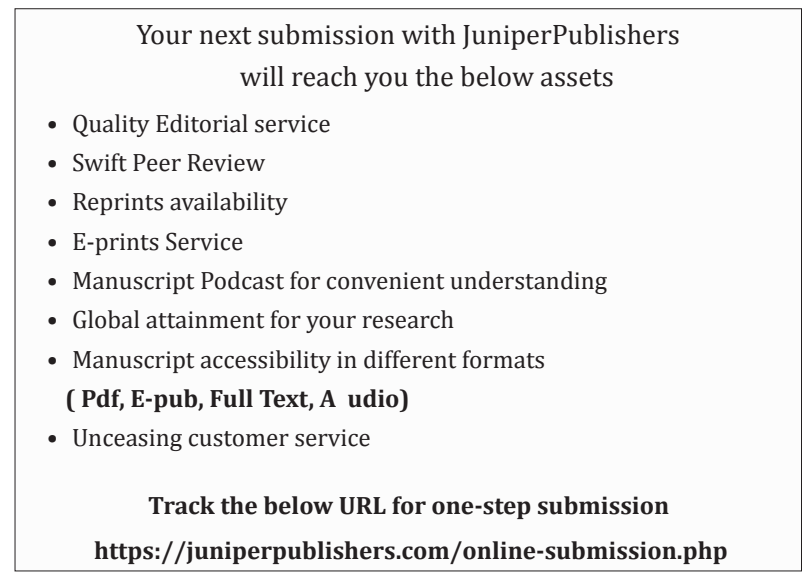

